-

LABORGESPRÄCHE 


\author{
RUDOLF GSCHWIND Und LUKAS ROSENTHALER \\ im Gespräch mit UTE HOLL
}

\title{
MIGRATION DER DATEN, ANALYSE DER BILDER, PERSISTENTE ARCHIVE
}

Prof. Dr. Rudolf Gschwind, Leiter des Imaging and Media Lab an der Universität Basel, und PD Dr. Lukas Rosenthaler, der ebenfalls zum leitenden Team des IML gehört und das Fach Informatik der philosophisch-historischen Fakultät koordiniert, forschen u. a. zu Techniken der Bewahrung audiovisuellen Materials. PEVIAN - Persistent Visual Archive heißt eines ihrer vom Schweizer Nationalfond finanzierten Projekte. Gschwind und Rosenthaler sind auch Mitglieder der Association of Moving Image Archivists.

$1 \mathrm{Vgl}$. Jeff Rothenberg, Digital Information Lasts Forever - Or Five Years, Whichever Comes First, RAND Video V-079, Leiden 1997

\section{U.H. Was bedeutet persistent in PEVIAR?}

R.G. Im Digitalen hat man den Vorteil, dass man Daten verlustfrei umkopieren kann. Es gibt sehr gute Methoden, eigentlich unbegrenzt Informationen aufzubereiten. Auf der anderen Seite aber ist da die Technik, und die ist extrem kurzlebig. Es gibt den schönen Spruch von Jeff Rothenberg: «Digital information lasts forever - or five years, whichever comes first. $\gg^{1}$ Das zentrale Problem in unserem Forschungsgebiet ist die Migration der Daten, das heißt, dass wir alle Jahre umkopieren müssen. Wir suchen nach Methoden, das zu umgehen. Aus der Vergangenheit gibt es im Wesentlichen zwei Archiviermethoden: einmal die Natur, genauer, der genetische Code. Das ist ganz einfach die Migration als automatischer Prozess. In einem anderen Projekt, dem DIST.AR.NET, Distributed Archival Network, hat Lukas Rosenthaler das untersucht. Die andere Methode schaut in die Geschichte der Medien; da gibt es zum Beispiel die Schrift, die auch digitale Daten darstellt. Das hat ja lange überlebt: in Büchern. Die Frage ist, inwiefern man Erfahrungen mit der Schrift ins digitale Zeitalter übertragen kann, so dass man nicht mehr migrieren muss. Dazu suchen wir ein Medium, das lange hält, also praktisch permanent ist. Heutige Medien sind relativ kurzlebig, für den Computer gemacht, schnell lesbar, aber nicht unbedingt 
hochstabil. Das ist der materiale Aspekt. Die zweite Frage hingegen wäre: Wie muss ich etwas archivieren, damit man das in Ioo Jahren zurücklesen kann?

\section{U.H. Man muss mit der Persistenz in den Futur II übergehen. Es wird so und so gelesen worden sein.}

R.G. Die Frage ist: Wie kann ich garantieren, dass der Archivar der Zukunft etwas zurücklesen kann, auch wenn nichts mehr da ist? Der Vergleich mit dem Buch ist wunderbar: Wir haben keine Technologie, der Mensch selber schaut auf die Schrift und kann dekodieren.

U.H. Man braucht kein Lesegerät, der Mensch ist selbst eines. Dazu gehört natürlich die Kulturtechnik des Lesens.

R.G. Das wäre die Formatfrage. Es klappt, wenn es sich um Sprachen handelt, die gelehrt und weiterverbreitet werden. Übertragen auf den Computer heißt langlebig: Man braucht ein Medium, das im Prinzip ohne Technologie gelesen werden kann. Das ist relativ schwierig. Wir kamen darauf, zur Speicherung ein visuelles Medium zu nutzen - deshalb Visual Archive. Wenn es in Zukunft Computer geben wird, dann auch digitale Kameras und Scanner. Das ist eine Basistechnologie. Ein permanentes Medium, das man gut kennt, ist der fotografische Film, der Mikrofilm, der eine hohe Datendichte verträgt; Das könnte ein kommerzieller Gesichtpunkt sein. Heutige Filme zeigen im Schnellalterungstest, dass sie einige Hundert Jahre halten. Das ist so gut wie permanent. Für uns interessant ist, dass man auf einem visuellen Träger Informationen in hybrider Form mischen kann: Auf Mikrofilm kann man digitale Information in Form von Punktemustern speichern, in zweidimensionalem Barcode, der dichtesten Speicherart, daneben aber auch einfach Text und Bild. Außerdem auch die Beschreibung davon, wie zurückzulesen ist, was die digitalen Daten bedeuten. Zunächst geht es im Projekt um technische Probleme: Wie dicht kann man schreiben, wie kann man die unausweichliche Alterung des Materials, die Beschädigungen, mit geeigneten Fehlerkorrekturen umgehen? Später kommen archivarische Aspekte dazu: Fotografische Filme, einmal belichtet und entwickelt, sind unveränderbar. Sobald man etwas verändert, sieht man das.

\section{U.H. Anders als beim digitalen Film.}

R.G. Beim digitalen Film sieht man Veränderungen nicht. Beim Mikrofilm sieht man sie mit bloßem Auge, mit einer Lupe vielleicht. Das eröffnet einen Aspekt der Authentifizierung im Digitalen. Für Anwendungen in der Filmarchivierung kann das interessant sein. Aber wenn man in Zeiträumen von etwa 50 Jahren rechnet, stellt sich vor allem die Frage: Wie kann ich die Beschreibung selbsterklärend machen, selfexplaining codes?

L.R. Das Dilemma digitaler Daten ist: Sie sind pure Information, immateriell, aber gebunden an einen materiellen Träger. 
U.H. Kann man denn sagen, ein Werk sei neutral gegenüber dem materiellen Träger?

L.R. Absolut. Das Material, auf dem die Daten registriert sind, spielt keine Rolle. Solange man den Code wieder decodieren kann. Im Prinzip sind digitale Daten ein Code symbolischer Zeichen. Im Computer werden nur zwei verwendet, o und I, aber im Prinzip ist das ein Alphabet mit zwei Buchstaben, dahinter eine Sprache. Wie bei einem Buch kann das auf Billigpapier stehen oder auf teurem oder in Stein gemeißelt sein ...

R.G. ... auf einem E-Book anzuschauen ...

U.H. Es gibt aber große Unterschiede darin, wie ein Film je nach Trägermaterial wirkt.

R.G. Das sind zwei Fragen, die nichts miteinander zu tun haben. Filmmaterial ist ein Datenträger, egal wofür. Das Speichermaterial ist Filmmaterial. Was man darauf speichert, ist gleichgültig: Musik oder Text, Datenbanken, Excel Sheets, digitalisierte Fotos, digitale Filme, MP4-Code ... Den kann ich auf eine CD oder ein Magnetband schreiben oder auf Mikrofilm. Wir nehmen fotografischen Film, um digitales Kino aufzubewahren. Man mag sich höchstens fragen, warum die Bilder überhaupt vorher digitalisiert werden. Warum nicht gleich die Bilder auf Film speichern, wie es ursprünglich ja schon war?

L.R. Wenn ein Film in digitaler Form vorliegt, stellt sich die Frage der Aufbewahrung. Das ist ein Problem, weil die elektronischen Datenspeicher nur für ganz kurze Aufbewahrungszeiten konzipiert sind: flüchtige Speicher. Die sollen Daten, als Faustregel, nicht länger als drei bis fünf Jahre bewahren.

U.H. Im Peviar werden analoge Filme digitalisiert, restauriert und gespeichert.

R.G./L.R. Das ist ein anderes Projekt ...

R.G. ... nämlich das der Rekonstruktion von Filmmaterial, das gealtert ist und Schäden genommen hat. Um einen ganzen Film zu rekonstruieren, ist es notwendig, szenenweise zu arbeiten. Wenn man einen Film einspannt und digitalisiert, wenn die Daten vorliegen und Algorithmen für die Rekonstruktion, kann man auch anhand der Daten die Szenen voneinander trennen, automatische Bildanalyse betreiben. Das haben wir bereits vor I 5 Jahren gemacht. Jede weitere Fragestellung geht über die Unterscheidung einzelner Szenen hinaus: Wie kann man aufgrund der Daten etwas über den Film aussagen, etwa über Schnittfolgen? Unsere Antworten waren rein technisch: Harte Schnitte sind relativ einfach zu erkennen, eine Überblendung schon schwieriger. Klar: Bildverarbeitung ist keine neue Wissenschaft. Das wird seit 50 Jahren praktiziert, früher im Bereich des Militärischen, des Medizinischen und der Raumfahrt. Das war extrem teuer, wurde aber häufig gemacht.

L.R. Ich unterscheide prinzipiell zwischen Bildverarbeitung und Bildanalyse. Bildverarbeitung heißt: Ich habe ein Bild, ändere etwas algorithmisch und be- 
komme wieder ein Bild. Bildanalyse heißt: Ich habe ein Bild, lasse einen Algorithmus über das Bild laufen, und am Schluss habe ich Information, die nicht mehr bildhaft ist. Bildanalyse ist sehr viel schwieriger, weil der Computer kein Konzept von Bild hat.

U.H. Die Frage ist, mit welchen Parametern man nach einem Bild fragt.

L.R. Genau. Für den Computer ist ein Bild ein Haufen ungeordneter Zahlen, die aussagen: An der Position X/Y habe ich die Helligkeit Z. Das ist kein Bild, aber der Computer sieht nur das. Das Problem ist, aus der Bildanalyse Rückschlüsse darauf zu ziehen, was in dem Bild sein könnte und welches der Unterschied zu einem anderen Bild ist. Bildverarbeitung, Bild zu Bild, ist nicht ganz einfach, aber relativ einfach. Bildanalyse jedoch, aus einem Bild automatisch Information zu gewinnen, ist für den Computer extrem schwierig zu programmieren, weil wir Menschen selbst nicht wissen, wie wir Bilder verarbeiten.

U.H. Durch Gestaltwahrnehmung beispielsweise ...

L.R. Die Gesetze der Gestalt, die wir seit den I92oer Jahren kennen, sind bis heute nicht in Algorithmen umgesetzt worden.

\section{U.H. Welche Möglichkeiten gibt es?}

L.R. Ich habe damit begonnen, das biologische Vorbild zu studieren, um zu verstehen, wie der Mensch Bilder analysiert. Aus der Wahrnehmungspsychologie und der Neurophysiologie gibt es Hinweise. Ich habe einmal solche Funktionsmodelle in Algorithmen umgesetzt und auf Bilder angewendet. Die Erkenntnis war, dass bestimme optische Täuschungen auf diese Weise erklärt werden können: Eine optische Täuschung ist ein Bild, in dem der Mensch etwas anderes sieht als im Bild drin ist. Daraus lernt man, wie die Algorithmen in Hirnprozessen funktionieren. Erstaunlich ist, dass es bis zu einem gewissen Punkt tatsächlich Algorithmen sind. Aber ab einem bestimmten weiteren Punkt weiß man nicht mehr, wie es funktioniert. Sobald es um Konzepte geht, wissen wir nicht, wie das Gehirn das macht. Das hat bisher noch niemand konsequent in Computeralgorithmen umgesetzt.

U.H. Es gibt also eine Punkt-für-Punkt-Übertragung von Daten, dann aber schwingt noch etwas mit wie - um ein Modell aus der Musik zu nehmen Subfrequenzen, die gar nicht da sind, die wir aber mithören.

L.R. Genau.

U.H. Gibt es ein vergleichbares Konzept für das Visuelle?

L.R. Nein, bis jetzt nicht. Ein Ansatzpunkt sind die Gestaltgesetze, mit denen man verstehen lernen könnte, wie das zum Beispiel im Vergleich zu den Subfrequenzen im Akustischen funktioniert. 
Sammlung obsoleter Speichermedien für Computer, Foto: Imaging \& Media Lab
U.H. Könnte man Parameter oder Algorithmen beschreiben, die im Digitalen etwas sichtbar machen, was es im Analogen nie gegeben hat?

R.G. Praktisch ja. In meinem Kurs zur Digitalfotografie habe ich festgestellt, dass es eine Gruppe Studierender gab, die im Sinne der Natürlichkeit des analogen Bildes fotografiert hat und versuchte, das Analoge nachzumachen, zum Beispiel schwarz-weiß zu machen. Eine andere Gruppe dagegen hat die Möglichkeiten der Bildmanipulation, die im Digitalen stecken, voll ausgenutzt. Insbesondere Farbmanipulation, die in der klassischen Fotografie extrem schwierig war. AuBerdem ist mir aufgefallen, dass in Ausstellungen die analogen Bilder gerahmt wurden, sauber im Passepartout, während die Digitalbilder mit einem Reißnagel an die Wand geheftet wurden. Damals sollte man das Gefühl bekommen, digital sei billig. Das kommt aus dem Drucker raus ...

U.H. ... nur eine Kopie, kein Original ...

R.G. ... da ist kein Bild, das hat man nicht erlebt, das hat eine Maschine gemacht.

L.R. Im Fernsehen haben digital gedrehte Serien eine eigene Ästhetik: Mir fiel bei der CSI-Serie auf, wie sehr mit künstlichen Farben gearbeitet wird. Die Farbsättigung wird ins Extreme getrieben, Farbfilter werden verwendet: oranger Himmel, total blaues Meer, wenn es CSI-Miami ist - das war früher beim Analogen schlicht nicht möglich.

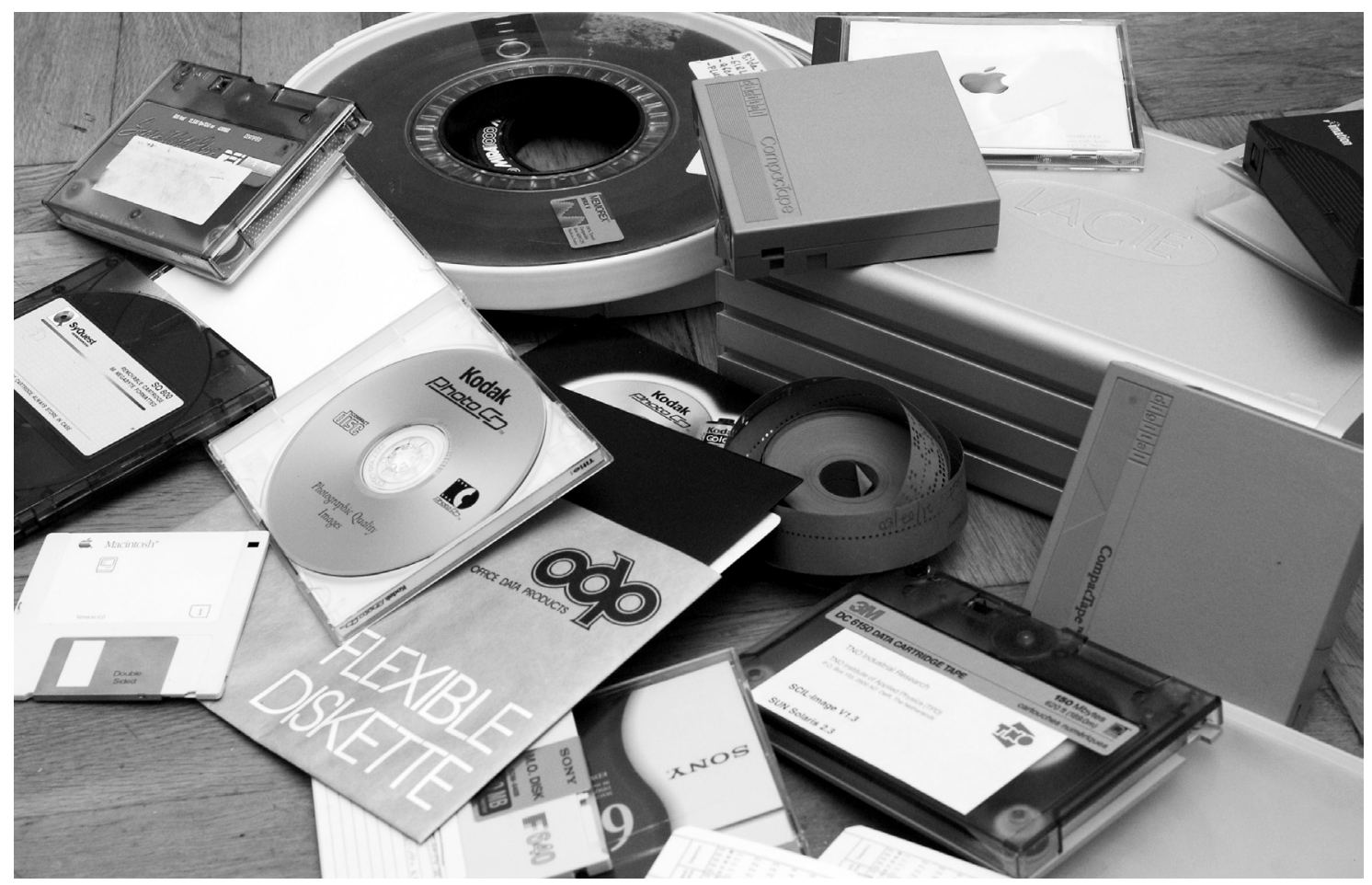


R.G. Zur Zeit stellt das Fensehen auf HD um, und alle Sender senden Testserien, durchweg hochfarbige Bilder, das Meer, die Blumen, die Pflanzen - die Farbe ist der große Unterschied zwischen dem Digitalen und dem Fotografischen. Die Farbgebung im fotografischen Film ist relativ undurchsichtig, ziemlich komplex; das liegt an der Chemie, die nicht gerade unverstanden, aber kaum beschreibbar ist. Im Fotolook bleiben die ungesättigten Farben ungesättigt und kippen auf einmal blitzschnell in gesättigte um. Das gibt es im Digitalen nicht, das geht graduell, da ist entweder alles gesättigt oder alles unsatt. Das ist ganz klar eine andere Ästhetik. Im Digitalen ist alles farbig.

U.H. Abwarten, im Video kann man inzwischen auch verschiedene Effekte über das Bild legen: Super-8- oder I6-mm-Anmutungen.

R.G. Und für den digitalen Fotoapparat kann man Ektrachromfilter kaufen, Kodakchromfilter, Tri-X-Filter, alles Retro. Im Inkjet findet man heute eine Vielzahl von Papieren, mehr als es in der Hochzeit der klassischen SchwarzWeiß-Fotografie je gegeben hat: Oberflächen in allen Sorten, matt, glänzend, halbglänzend, was Sie wollen. Der letzte Schrei ist Baryt-Inkjet-Papier. Spannend, wie die Welt zurück in die klassische Fotografie will.

L.R. Spannend auch für die Restauration alter Kinofilme. Da geraten wir dann ins Ästhetikproblem: Ich digitalisiere einen alten Film, weil ich ihn restaurieren möchte, und habe darin alle Freiheiten. Aber was mache ich damit? Eine ethische Frage, die heute jeder Restaurator, der mit digitalen Methoden arbeitet, beantworten muss. Technicolor zum Beispiel hat eine ganz eigene Farbästhetik. Will man die im Digitalen behalten oder meint man, das liegt nur daran, dass Technicolor keine gute Technik war - man hätte das damals auch anders gemacht, wenn man es denn hätte machen können!

\section{U.H. Also ist der materielle Träger doch nicht indifferent gegenüber dem Ma- terial?}

L.R. Das war auf der Konferenz in St. Louis ${ }^{2}$ anlässlich eines restaurierten Films ein großes Thema. In Technicolor ist der Rotkanal immer unscharf, aus technischen Gründen. Ein in St. Louis vorgestelltes Projekt hat den Rotkanal speziell geschärft, das heißt: Jener Saum, der typisch ist für die harten Kanten im Technicolor, war bei der Restauration verschwunden. Es gab eine zweistündige Diskussion, ob man das darf oder nicht. Im Digitalen kann man beides: eine historisch authentische Rekonstruktion oder eine, die einer modernen Ästhetik genügt.

U.H. Wobei authentisch für die Wahrnehmung eine mediale Frage ist: Die Filme aus den 7 oer Jahren sehen heute rötlicher aus als sie in den Siebzigern waren.

L.R. Ja, aber ${ }_{1} 983$ bereits hat Ruedi Gschwind - ich bin ${ }_{9} 985$ dazugestoßen - eine Methode gefunden, die nicht perfekt ist, aber erlaubt, die Originalfarbeindrücke wiederzugeben. Sie basiert auf der Idee, den chemischen Verfall algorithmisch
2 Jahrestagung der Association of Moving Image Archivists 2009 in St. Louis. 
Filmrollen mit starkem Essigsäure-Syndrom, Foto: Imaging \& Media Lab zu simulieren. Wenn man das kann, kann man auch die Uhr zurückdrehen, denn man simuliert nicht den Farbeindruck, sondern die Chemie. Hierzu gab es ein interessantes Erlebnis mit einem Basler Afrikaforscher, Professor Gardi, der in den 5 oer Jahren sehr viele Farbfotos und Filme gemacht hat. Bei einem Foto sind wir fast verzweifelt, weil die Afrikaner, die dort abgebildet waren, bei einer guten Rekonstruktion blaue Haut hatten. Dann kam der alte Gardi ins Labor und ist völlig ausgeflippt, weil er gesagt hat, dieser Stamm, der heute ausgestorben ist, hätte genau so blaue Haut gehabt.

\section{U.H. Nicht nur auf dem damaligen Filmmaterial?}

L.R. Nein, auch in Wirklichkeit!

R.G. Das war einer dieser Stämme, die so eine stahlblaue Haut hatten.

L.R. Das war für uns der Hinweis, dass diese Rekonstruktionsmethode im Rahmen des Möglichen relativ objektive Resultate ergab. Wir haben auch Tests gemacht mit Agfa-Filmen von 1938, da haben die Rekonstruktionen nicht gut ausgesehen, weil die Filme damals auch nicht gut ausgesehen haben! Die Methode funktioniert eigentlich recht gut.

U.H. Weil die chemische Formel die Norm darstellt und nicht die menschliche Wahrnehmung. Kommen wir zurück zur Konferenz: Da ging es nicht nur um Filmmaterial, das gesichert und archiviert werden muss, sondern auch um Videoformate.

L.R. Bei den Videoformaten ist die Situation schlicht dramatisch. Alle alten Formate bis hin zu U-matic können eigentlich nicht mehr gerettet werden.

U.H. Das umfasst ca. zehn Jahre Fernsehzeit ...

L.R. Mehr! Das geht von den 2-Zoll-Bändern der frühen 5oer Jahren bis zum U-matic der 7oer Jahre. Alles eigentlich verloren!

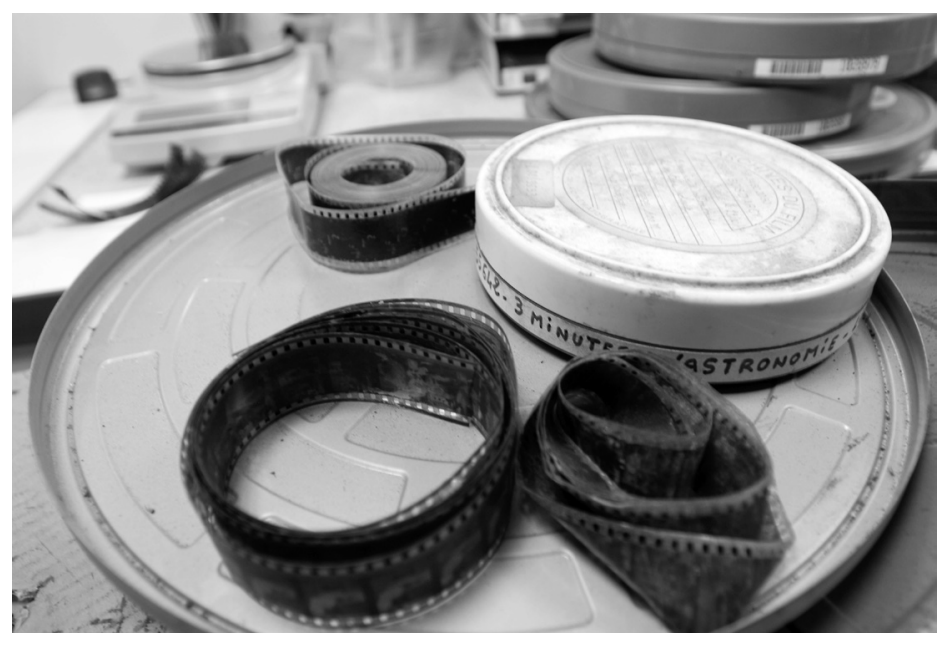

U.H. Im Prinzip die populärste Kultur dieser Zeit ...

L.R. Ja! Ist nicht mehr zu sehen! Das Problem ist, dass die Datenträger, die Bänder, zwar zum Teil noch brauchbar sind, aber die Geräte nicht mehr. Diese Geräte können heute nicht mehr gebaut werden, weil die gesamte Technologie ringsherum fehlt. Es gibt im Moment noch einen Betrieb in der Welt, der Ersatzmagnetköpfe für diese alten Videogeräte herstellt. Das ist eine Kleinfirma, und derjenige, der sie baut, ist jetzt 75 Jahre alt. 
Wenn dieser Mann stirbt oder aufhört zu arbeiten, dann werden innerhalb von ein bis zwei Jahren sämtliche Geräte dieser Art nicht mehr funktionieren, weil die Magnetköpfe ersetzt werden müssten.

U.H. Es gibt Tausende von Fernsehanstalten, die Interesse daran haben sollten, die Geräte zu erhalten. Warum legt man das nicht neu auf?

L.R. Das ist zu teuer. Die Produktion effektiv wieder in Gang zu setzen, würde Millionen kosten. Denn diese Technologie ist verknüpft mit anderen Technologien aus jener Zeit. Zum Beispiel: Zur Herstellung dieser Magnetköpfe wird ein spezielles Magnetband benötigt, um diese zu justieren. Dieses Magnetband wurde von der Firma $3 M$ hergestellt, die nur noch zehn Meter von diesem Band hat. Das wird gerade verbraucht, weil es sich abnützt. Auf die Frage, was es kosten würde, dieses Magnetband mit speziellen Markierungen wieder herzustellen, gab $3 M$ einen Millionenbetrag in US-Dollar durch, nur zur Herstellung einer winzigen Komponente. Alles in allem geht es um dreistellige Millionenbeträge, die benötigt würden, um diese Geräte wieder zu bauen.

U.H. Gibt es denn von Ihrer Seite irgendeine Intervention in den Videobereich, oder müssen Sie auch abwarten, bis jemand das finanziert?

L.R. Wir haben auch hier Ideen gehabt, die an mehreren Problemen gescheitert sind: Einerseits sind wir hier am Institut zu klein, andererseits fehlt in der Schweiz die entsprechende Industrie. Es gab offensichtlich ein EU-Projekt, einen Prototyp in dieser Richtung zu bauen, das jedoch nicht weiter verfolgt wurde. Mit unserer eigenen Idee kommen wir wieder auf das Visuelle zurück. Wir wollten das Magnetfeld auf dem Magnetband sichtbar machen und das mit einer digitalen Kamera abfilmen. Der Rest lässt sich dann über normale Computerprogramme machen. Das Problem ist, wie ein Magnetfeld sichtbar zu machen wäre. Wir haben verschiedene Ideen gehabt und Tests mit magnetischen Flüssigkeiten gemacht.

R.G. Mit magnetischen Flüssigkristallen.

U.H. Da die Zeilen auf dem Videoband nicht mehr gelesen werden können, weil die Videoköpfe als Lesegeräte fehlen, war Ihre Idee, direkt auf die Codierung zu schauen ...

L.R. ... um das Magnetfeld sichtbar zu machen und dann mit einer Kamera zu lesen. Dann würde die Bildanalyse dahinter geschaltet und so das Videosignal wieder aufgearbeitet.

\section{U.H. Video im Futur II.}

R.G. Schwierig. Keine triviale Angelegenheit ...

L.R. Meine Idee war: Es gibt einen physikalischen Effekt, den sogenannten KerrEffekt, mit dem man per Laser Magnetfelder sichtbar machen könnte. Das würde ich gerne verfolgen, aber dazu müssen wir natürlich mit der Physik zusammenarbeiten. 
U.H. Man muss davon ausgehen, dass gerade 20 Jahre Populärkultur verloren gehen.

L.R. Jim Lindner, einer der Väter der Magnetbänder, hat auf der Tagung in St. Louis einen deprimierenden Vortrag gehalten. Erschreckend. Von diesem Teil der Populärkultur wird nur noch ein winziger Prozentsatz erhalten bleiben, das Meiste ist schon weg, zwar noch physisch da, als Material, aber das kann nicht mehr gelesen, das Bild kann nicht mehr reproduziert werden. In Europa gibt es vermutlich nur noch weniger als ein Dutzend Geräte, die 2-Zoll-Bänder abspielen können.

\section{U.H. Und die Kinoproduktion?}

R.G. Da wird das Gleiche passieren wie in der Fotografie: In ein paar Jahren ist das Kino filmlos.

L.R. Wir haben den Vertreter von Kinoton, dem letzten großen Hersteller von analogen Produktionsapparaten, in St. Louis getroffen, der sagte, dass analoge Projektoren demnächst nicht mehr produziert werden. Ökologischer ist das auf jeden Fall, anstatt Tausende von Projektionskopien herzustellen, die eine Woche nach Kinostart wieder weggeworfen werden. Es ist ökologischer und ökonomischer. Der einzige Widerstand kommt von den Kinobetreibern, da die Umstellung mit Kosten verbunden ist.

R.G. Digital ist das Kino ja schon lange, aber es wird filmlos werden. Das Fernsehen wurde sehr schnell auf HD umgestellt; mit Blue-Ray, HD, Beamer oder Großbildschirm hat man zu einem vergleichsweise niedrigen Preis Superqualität. Um Menschen wieder ins Kino zu bringen, gibt es jetzt 3D, wie immer in der Kinogeschichte bei technischen Umstellungen. Das 3D-Verfahren funktioniert im Fernsehen noch nicht. Mit 3D können die Kinobetreiber gezwungen werden, auf digital umzustellen, wenn sie mit 2D kein Geld mehr verdienen, denn dieses Verfahren zieht das Publikum wieder an. Danach verschwindet 3D wieder in der Versenkung bis zu einer Wiederauferstehung in x Jahren ... Kondratjeff-Zyklen, in der Wirtschaft wie im Kino. I 840 die Erfindung der Fotografie, I 890 das Industrieprodukt und der Kinofilm, I 940 das Fernsehen, I990 das Digitale, was wird wohl 2040 sein? Vielleicht kann man dann das innere Bild anzapfen oder das sehen, was man träumt ...

U.H. ... wie es in Avatar in 3D vorgestellt wird, das Rückenmark lässt sich mit natürlichen Informationssystemen verknüpfen.

R.G. Cyberspace ...

L.R. ... Immersive Virtual Realities ... in diese Richtung könnte es gehen.

R.G. Wenn ich das wüsste, müsste ich nicht mehr forschen, das wäre ja langweilig. 